\title{
Daily Reportable Disease Spatiotemporal Cluster Detection, New York City, New York, USA, 2014-2015
}

\section{Sharon K. Greene, Eric R. Peterson, Deborah Kapell, Annie D. Fine, Martin Kulldorff}

Each day, the New York City Department of Health and Mental Hygiene uses the free SaTScan software to apply prospective space-time permutation scan statistics to strengthen early outbreak detection for 35 reportable diseases. This method prompted early detection of outbreaks of community-acquired legionellosis and shigellosis.

$\mathrm{T}$ he Bureau of Communicable Disease (BCD) at the New York City Department of Health and Mental Hygiene (DOHMH) monitors and investigates $>70$ reportable diseases among the city's 8.49 million residents. Each day, healthcare providers and laboratories submit $\approx 1,000$ communicable disease reports to BCD. Clusters (significant increases in observed vs. expected cases) and outbreaks (clusters believed to be associated with a common infection source) are detected through several methods, including notification by astute healthcare providers and by applying the modified historical limits method to detect increases in disease counts during the previous 4 weeks (1). This temporal analysis is applied weekly citywide and for each of 5 boroughs and 42 neighborhoods.

Cluster detection methods have been applied to syndromic data sources (e.g., emergency department visits) since the early 2000 s $(2,3)$. Less extensively described is cluster detection using reportable disease data, which reflect specific laboratory-confirmed diagnoses, contain patient home addresses, and may include illness onset dates and work addresses collected during patient interviews and medical record reviews. Other public health practitioners have applied purely temporal prospective cluster detection methods to reportable disease data $(4,5)$ or conducted proof-of-concept spatiotemporal prospective analyses $(6,7)$. However, published descriptions of actual prospective application of spatiotemporal methods to reportable diseases are rare $(8,9)$, suggesting lack of wide-

Author affiliations: New York City Department of Health and Mental Hygiene, Queens, New York, USA (S.K. Greene,

E.R. Peterson, D. Kapell, A.D. Fine); Brigham and Women's Hospital, Harvard Medical School, Boston, Massachusetts, USA (M. Kulldorff)

DOI: http://dx.doi.org/10.3201/eid2210.160097 spread adoption among public health officials. We describe BCD's experience with automated daily reportable disease spatiotemporal cluster detection using prospective spacetime permutation scan statistics (3) in SaTScan (10) during February 2014-September 2015, highlighting instances in which findings guided public health action.

\section{The Study}

For 35 reportable communicable diseases for which cluster detection could inform programmatic activities (1), we analyzed disease counts for patients of all ages combined. For amebiasis, cryptosporidiosis, and giardiasis, for which outbreaks among young children are of particular interest, additional analyses were restricted to disease counts among patients $<5$ years of age, for 38 total daily analyses.

In BCD's application, the space-time permutation scan statistic detects disease clusters in space-time cylinders centered on every census tract centroid; the circular base represents space (maximum geographic cluster size of $50 \%$ of all reported cases), and the height represents time (maximum temporal window length of 30 days, for most diseases). For each cylinder, a likelihood ratio-based test statistic is calculated. The test statistic is considered elevated if the observed disease count during the time window in census tracts with centroids inside the cylinder's circular base exceeds the expected number of cases, which is a function of 1) the case count in the circle during a baseline period (which accounts for any purely geographic variations in disease occurrence, diagnosis, and reporting) and 2) the total case count citywide during the time window (which accounts for citywide purely temporal patterns, such as seasonality or secular trends) (3). The cylinder with the maximum test statistic is the cluster least likely to be due to chance under the null hypothesis that the same process generated disease counts inside and outside the cylinder.

To create a simulated dataset, cases' dates are randomly shuffled and assigned to the original census tracts. The maximum statistic for each simulated dataset is calculated in the same way as for the observed dataset. For each disease, this process is repeated daily 999 times. The maximum value for the observed dataset is ranked among the 999 trial maxima. A p value (range 0.001-1) is derived from this ranking; $p=0.001$ represents the highest significance relative to the permutation trials. The Monte Carlo approach to deriving significance by using repeated trials, 
each permuting observed data attributes, is designed to control for multiple testing.

A recurrence interval (RI) is calculated as the reciprocal of the $p$ value and represents the number of days of daily surveillance required for the expected number of clusters at least as unusual as the observed cluster to be equal to 1 by chance (11). We defined a signal as any cluster with an $\mathrm{RI} \geq 100$ days; that is, during any 100 -day daily analysis period, the expected number of clusters at least as unlikely as the current cluster is 1 .

We developed a SAS program (SAS Institute, Inc., Cary, NC, USA) to generate case and parameter files (Table 1), read in a coordinate file of census tract centroids, invoke SaTScan in batch mode, read analysis results back into SAS for further processing, and output files to secured folders. For any signals, the program also generated emails notifying BCD leadership and staff responsible for followup (online Technical Appendix, http://wwwnc.cdc.gov/ EID/article/22/10/16-0097-Techapp1.pdf).

This automated analysis detected the second largest US outbreak of community-acquired legionellosis (12), identifying a cluster of 8 cases centered in the South Bronx on Friday, July 17, 2015 (RI $=500$ days) (Figure), before any human public health monitor noticed it. On Monday, July 20, an increase in cases was independently noticed by BCD staff members routinely investigating individual cases, and on July 21, an infection-control nurse working in the outbreak area called BCD to report an increase. The DOHMH and state and federal partners conducted an extensive epidemiologic, environmental, and laboratory investigation to identify and remediate the outbreak source, a cooling tower.

A shigellosis outbreak among the observant Jewish community in Brooklyn (13) began in late October 2014 and was detected with 9 cases on November 14, 2014 $(\mathrm{RI}=333$ days $)$. BCD does not routinely investigate individual shigellosis reports, so automated analysis alone prompted early outbreak identification. Shigellosis outbreaks within this community occur cyclically and have been linked to daycare and preschool attendance (14). Starting in mid-November, BCD staff visited community schools, daycare centers, and health fairs to promote appropriate handwashing. The outbreak subsided by midMarch 2015. Other clusters prompting investigations

Table 1. Case file specifications for routine reportable disease analyses in New York City, New York, using the prospective spacetime permutation scan statistic

\begin{tabular}{llc}
\hline Feature & Selection & Notes \\
\hline Geographic & Census tract (defined using US Census & The less data are spatially aggregated, the more precisely areas with
\end{tabular}

aggregation 2000 boundaries) of residential address elevated rates can be identified. New York City has 2,216 census tracts at time of report* in an area of 305 square miles.

Date of interest Event date, defined using hierarchy of Defining reportable disease clusters according to when case-patients for analysis onset date $\rightarrow$ diagnosis date (collection became ill is preferred. However, onset date is missing for most casedate of first specimen testing positive $\rightarrow$ patients who have not yet been interviewed, and each case needs a date report date $\rightarrow$ date event created in to be included in analysis. Thus, the best available proxy for onset date is surveillance database used. Because we use daily data (rather than weekly, monthly, or yearly data), the time precision is specified as day on the SaTScan (http://www.satscan.org/) input tab. The time precision parameter indicates the temporal resolution of the data in the case file.

Study period $\quad 1 \mathrm{y}$ for most diseases, ending the day before analysis $†$

One year is a reasonable choice, balancing the need for a period long enough to establish a stable local baseline for each spatial unit, yet short enough to avoid variable secular trends (e.g., geographically different increases in the underlying population of a spatial unit). Analyses are run each morning using data with event dates through the previous day.

Case inclusion Include all reported cases, regardless of criteria current status (e.g., confirmed, probable, suspected, pending, noncase) $\dagger$ Depending on the disease, cases initially might be assigned a transient pending status and, upon investigation, be reclassified as a case (confirmed, probable, or suspected) or a noncase. Timeliness is preserved by analyzing all reported cases, including noncases and pending cases, regardless of whether they ultimately will be confirmed. By analyzing all reported cases, case inclusion criteria are consistent across the study period. If instead the case file were restricted to confirmed and pending cases, then analyses would be biased toward

false signaling, as some cases with an initial pending status will be ultimately reclassified after investigation as a noncase. This reclassification process is complete for the baseline but ongoing for the current period of interest (1), and the speed of reclassification might vary geographically.

\begin{tabular}{lcc}
\hline $\begin{array}{l}\text { Day-of-week } \\
\text { variable }\end{array}$ & $\begin{array}{c}\text { Include a variable that indicates the day } \\
\text { of the week (1-7) }\end{array}$ & $\begin{array}{c}\text { The analysis automatically adjusts for day-of-week effects but not for } \\
\text { space by day-of-week interaction. Including this variable in the SaTScan } \\
\text { case file accounts for how the daily pattern of health-seeking behavior } \\
\text { and diagnoses might vary geographically. }\end{array}$
\end{tabular}
*Exception to residential address at time of report: if the residential address is not geocodable (e.g., because the case-patient is not a resident of the city or because a post office box is reported instead of a street address), then the geocoded work address, if available, is substituted. †For exceptions, see online Technical Appendix (http://wwwnc.cdc.gov/EID/article/22/10/16-0097-Techapp1.pdf). 


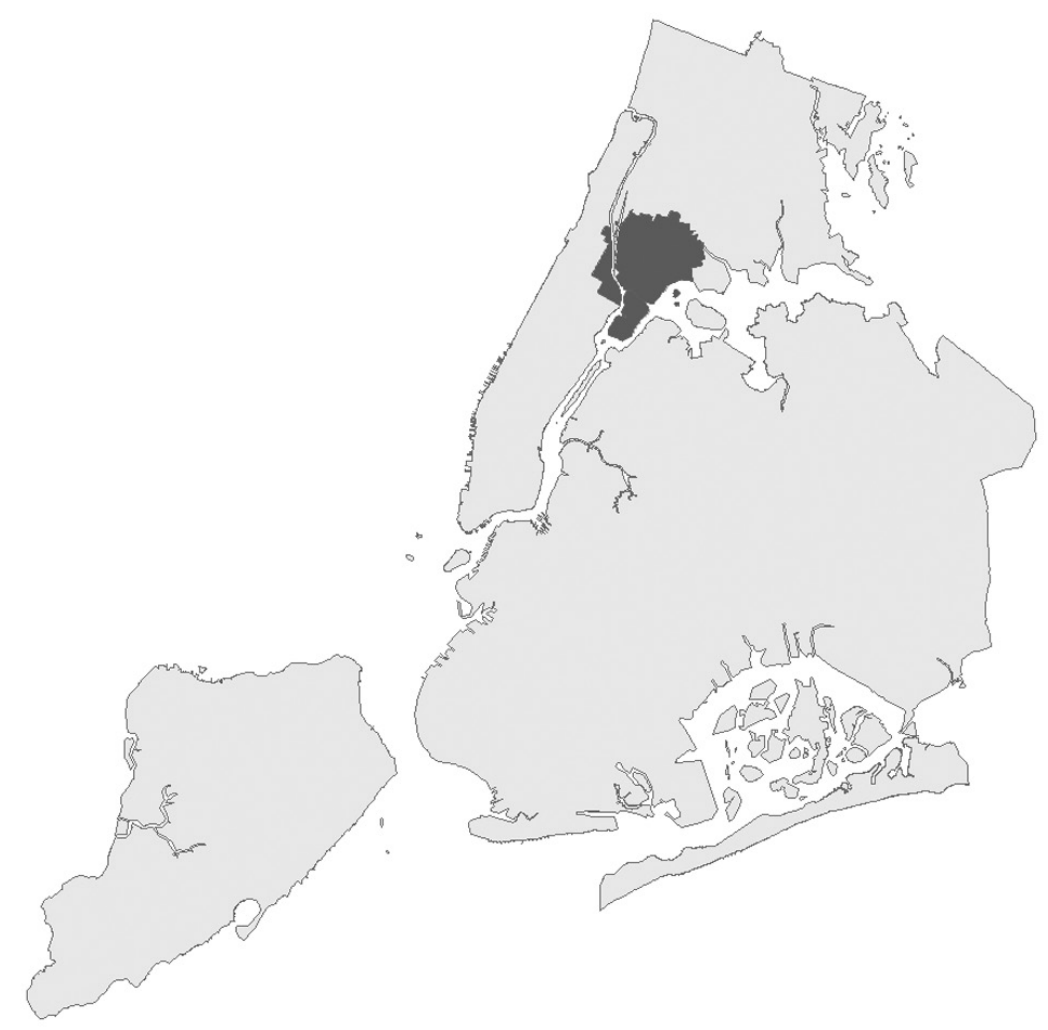

Figure. Automated output from spatiotemporal analysis on July 17,2015 , indicating a cluster (dark gray) of 8 legionellosis cases over 8 days centered in the South Bronx, New York City, New York, USA. In subsequent days, this cluster expanded in space and time into the second largest US outbreak of community-acquired legionellosis.

included legionellosis (Queens, April-May 2015) and campylobacteriosis (Brooklyn, October 2014). During a 1 -year period, 28 unique signals were observed across 15 diseases (Table 2), which staff perceived as a reasonable number for investigation.

Not all detected clusters were actionable. No public health response was conducted for an amebiasis cluster (Manhattan, April 2015; RI = 143 days) consisting of 6 men (34-49 years of age) diagnosed within a 12-day period and residing within a 0.35 -mile radius because no casepatients were identified as food handlers or daycare workers. A public health response also was not conducted for a giardiasis cluster (Bronx, April 2015; RI $=1,000$ days) that consisted of 6 household members who acquired the infection during international travel. Investigators were interested in being notified of and following such clusters over time, even if they ultimately were not actionable or verified as true outbreaks.

\section{Conclusions}

Several outbreaks in New York City, New York, were detected by daily automated spatiotemporal analyses. Early cluster detection facilitated prioritization of individual case investigations, outbreak recognition and investigation, provider and community outreach, and timely intervention to limit sickness and death. This method has proven particularly useful for identifying and monitoring outbreaks of shigellosis $(6,8,9)$ and legionellosis and might be useful for monitoring additional diseases with outbreak potential, including pertussis, syphilis, and tuberculosis.

Key to the system's success is a strong informatics infrastructure, especially electronic laboratory reporting and near real-time geocoding of surveillance data. Other facilitators include a powerful statistical disease surveillance methodology, knowledgeable epidemiologists to interpret signals, and adequate outbreak investigation resources.

These methods could be useful to other health departments receiving more reports than can be rapidly reviewed manually. State health departments could consider conducting similar analyses to detect clusters spanning multiple jurisdictions.

\section{Acknowledgments}

We thank Alison Levin-Rector for contributions to the SAS code and Lisa Alleyne, Catherine Dentinger, Robert Fitzhenry, Lucretia Jones, Lan Li, Ellen Lee, Sally Slavinski, Vasudha Reddy, HaeNa Waechter, and Don Weiss for contributions to signal interpretations for particular diseases.

S.K.G., E.R.P., and D.K. were supported by the Public Health Emergency Preparedness Cooperative Agreement (grant 5U90TP000546-03) from the Centers for Disease Control and Prevention. A.D.F. was supported by New York City tax levy funds. M.K. was funded by the National Institutes of Health (grant RO1CA165057). 
Table 2. Signaling rates at 3 recurrence interval thresholds for 35 reportable diseases under surveillance in New York City, New York, USA, 2014-2015*

\begin{tabular}{|c|c|c|c|c|}
\hline \multirow[b]{2}{*}{ Disease } & \multirow[b]{2}{*}{$\begin{array}{l}\text { Annual no. } \\
\text { cases } \ddagger\end{array}$} & \multicolumn{3}{|c|}{ No. signals during $365 \mathrm{~d}$ of prospective surveillance $\dagger$} \\
\hline & & $\begin{array}{c}\text { Recurrence interval } \\
\geq 365 \mathrm{~d} \S\end{array}$ & $\begin{array}{c}\text { Recurrence interval } \\
\geq 100 \mathrm{~d}\end{array}$ & $\begin{array}{l}\text { Recurrence interval } \\
\geq 30 \mathrm{~d}\end{array}$ \\
\hline Amebiasis & 476 & 0 & 1.2 & 4.3 \\
\hline Babesiosis & 57 & 0 & 0 & 0 \\
\hline Campylobacteriosis & 1,663 & 0.6 & 0.6 & 4.9 \\
\hline Chikungunya & 171 & 0.6 & 1.8 & 3.1 \\
\hline Cholera & 0 & 0 & 0 & 0 \\
\hline Cryptosporidiosis & 135 & 0 & 0 & 0.6 \\
\hline Cyclosporiasis & 51 & 0 & 0 & 1.2 \\
\hline Dengue & 57 & 0 & 0 & 1.8 \\
\hline Encephalitis & 2 & 0 & 0 & 0 \\
\hline Giardiasis & 871 & 1.2 & 1.8 & 5.5 \\
\hline Hemolytic uremic syndrome & 4 & 0 & 0 & 0 \\
\hline Hepatitis A & 78 & 1.9 & 1.9 & 5.8 \\
\hline Acute hepatitis B & 51 & 0.6 & 1.2 & 3.7 \\
\hline Hepatitis D & 0 & 0 & 0 & 0 \\
\hline Hepatitis E & 0 & 0 & 0.6 & 0.6 \\
\hline Human granulocytic anaplasmosis & 51 & 0.6 & 0.6 & 0.6 \\
\hline Human monocytic ehrlichiosis & 8 & 0 & 0.6 & 0.6 \\
\hline Invasive group A Streptococcus disease & 263 & 0 & 0 & 1.8 \\
\hline Invasive group B Streptococcus disease & 33 & 0.6 & 1.2 & 2.4 \\
\hline Invasive Haemophilus influenzae disease & 97 & 0 & 0 & 1.8 \\
\hline Invasive Streptococcus pneumoniae disease & 647 & 0 & 1.2 & 1.8 \\
\hline Legionellosis & 434 & 9.1 & 9.1 & 11.4 \\
\hline Listeriosis & 34 & 0 & 0 & 0.6 \\
\hline Malaria & 187 & 0.6 & 1.8 & 4.3 \\
\hline Meningococcal disease & 8 & 0 & 0 & 0.6 \\
\hline Noncholera Vibrio spp. infection & 18 & 0 & 0 & 0 \\
\hline Paratyphoid fever & 11 & 0 & 0 & 0 \\
\hline Rickettisalpox & 9 & 0 & 0 & 0 \\
\hline Rocky Mountain spotted fever & 6 & 0 & 0 & 2.4 \\
\hline Shiga toxin-producing Escherichia coli & 96 & 0 & 0 & 0 \\
\hline Shigellosis & 806 & 1.8 & 1.8 & 6.1 \\
\hline Typhoid fever & 31 & 0 & 1.9 & 3.8 \\
\hline $\begin{array}{l}\text { Vancomycin-intermediate Staphylococcus } \\
\text { aureus infection }\end{array}$ & 28 & 0 & 0 & 0 \\
\hline West Nile virus disease & 19 & 0 & 0 & 0 \\
\hline Yersiniosis & 25 & 0 & 0 & 0 \\
\hline $\begin{array}{l}\text { Total signals across all diseases under } \\
\text { surveillance }\end{array}$ & NA & 17.8 & 27.6 & 69.8 \\
\hline \multicolumn{5}{|c|}{ 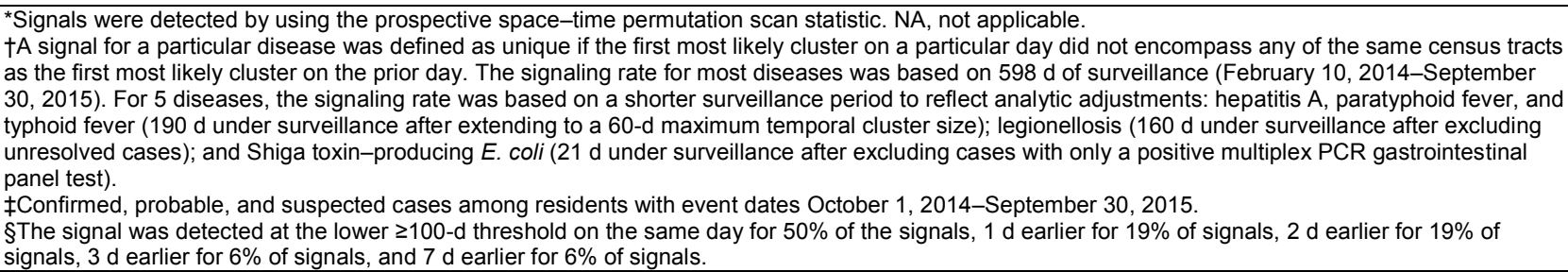 } \\
\hline
\end{tabular}

SaTScan is a trademark of Martin Kulldorff. The SaTScan software was developed under the joint auspices of Martin Kulldorff, the National Cancer Institute, and Farzad Mostashari of the New York City Department of Health and Mental Hygiene.

Dr. Greene is director of the Data Analysis Unit at the Bureau of Communicable Disease of the New York City Department of Health and Mental Hygiene, Queens, New York. Her research interests include infectious disease epidemiology and applied surveillance methods for outbreak detection.

\section{References}

1. Levin-Rector A, Wilson EL, Fine AD, Greene SK. Refining historical limits method to improve disease cluster detection, New York City, New York, USA. Emerg Infect Dis. 2015;21:265-72. http://dx.doi.org/10.3201/eid2102.140098

2. Heffernan R, Mostashari F, Das D, Karpati A, Kulldorff M, Weiss D. Syndromic surveillance in public health practice, New York City. Emerg Infect Dis. 2004;10:858-64. http://dx.doi.org/10.3201/eid1005.030646

3. Kulldorff M, Heffernan R, Hartman J, Assuncao R, Mostashari F. A space-time permutation scan statistic for disease outbreak detection. PLoS Med. 2005;2:e59. http://dx.doi.org/10.1371/journal.pmed.0020059 
4. Hutwagner L, Thompson W, Seeman GM, Treadwell T. The bioterrorism preparedness and response Early Aberration Reporting System (EARS). J Urban Health. 2003;80(Suppl 1):i89-96.

5. Rigdon SE, Turabelidze G, Jahanpour E. Trigonometric regression for analysis of public health surveillance data. Journal of Applied Mathematics. 2014;2014:1. http://dx.doi.org/10.1155/2014/673293

6. Jones RC, Liberatore M, Fernandez JR, Gerber SI. Use of a prospective space-time scan statistic to prioritize shigellosis case investigations in an urban jurisdiction. Public Health Rep. 2006;121:133-9.

7. Hughes GJ, Gorton R. An evaluation of SaTScan for the prospective detection of space-time Campylobacter clusters in the North East of England. Epidemiol Infect. 2013;141:2354-64. http://dx.doi.org/10.1017/S0950268812003135

8. Viñas MR, Tuduri E, Galar A, Yih K, Pichel M, Stelling J, et al. Laboratory-based prospective surveillance for community outbreaks of Shigella spp. in Argentina. PLoS Negl Trop Dis. 2013;7:e2521. http://dx.doi.org/10.1371/journal.pntd.0002521

9. Glatman-Freedman A, Kaufman Z, Kopel E, Bassal R, Taran D, Valinsky L, et al. Near real-time space-time cluster analysis for detection of enteric disease outbreaks in a community setting. J Infect. 2016;73:99-106. http://dx.doi.org/10.1016/j.jinf.2016.04.038

10. Kulldorff M; Information Management Services, Inc. SaTScan v9.1.1: software for the spatial and space-time scan statistics. 2015 [cited 2015 Sep 24]. http://www.satscan.org/
11. Kleinman K, Lazarus R, Platt R. A generalized linear mixed models approach for detecting incident clusters of disease in small areas, with an application to biological terrorism. Am J Epidemiol. 2004;159:217-24. http://dx.doi.org/10.1093/aje/ kwh029

12. New York City Department of Health and Mental Hygiene. Health Alert Network. 2015 alert 21: increase in Legionnaire's disease in the Bronx [cited 2015 Sep 24]. https://a816-health30ssl.nyc.gov/sites/nychan/Lists/AlertUpdateAdvisoryDocuments/HAN_LegionellaSouthBronx.pdf

13. New York City Department of Health and Mental Hygiene. 2014 Alert 39: Outbreak of shigellosis in Borough Park and Williamsburg [cited 2015 Sep 24].

https://a816-health30ssl.nyc.gov/sites/nychan/Lists/AlertUpdateAdvisoryDocuments/HAN_Shigella.pdf

14. Garrett V, Bornschlegel K, Lange D, Reddy V, Kornstein L, Kornblum J, et al. A recurring outbreak of Shigella sonnei among traditionally observant Jewish children in New York City: the risks of daycare and household transmission. Epidemiol Infect. 2006;134:1231-6. http://dx.doi.org/10.1017/S0950268806006182

Address for correspondence: Sharon K. Greene, New York City Department of Health and Mental Hygiene, 42-09 28th St, CN 22A, WS 06-154, Queens, NY 11101, USA; email: sgreene4@health.nyc.gov

\section{April 2015: Emerging Viruses}

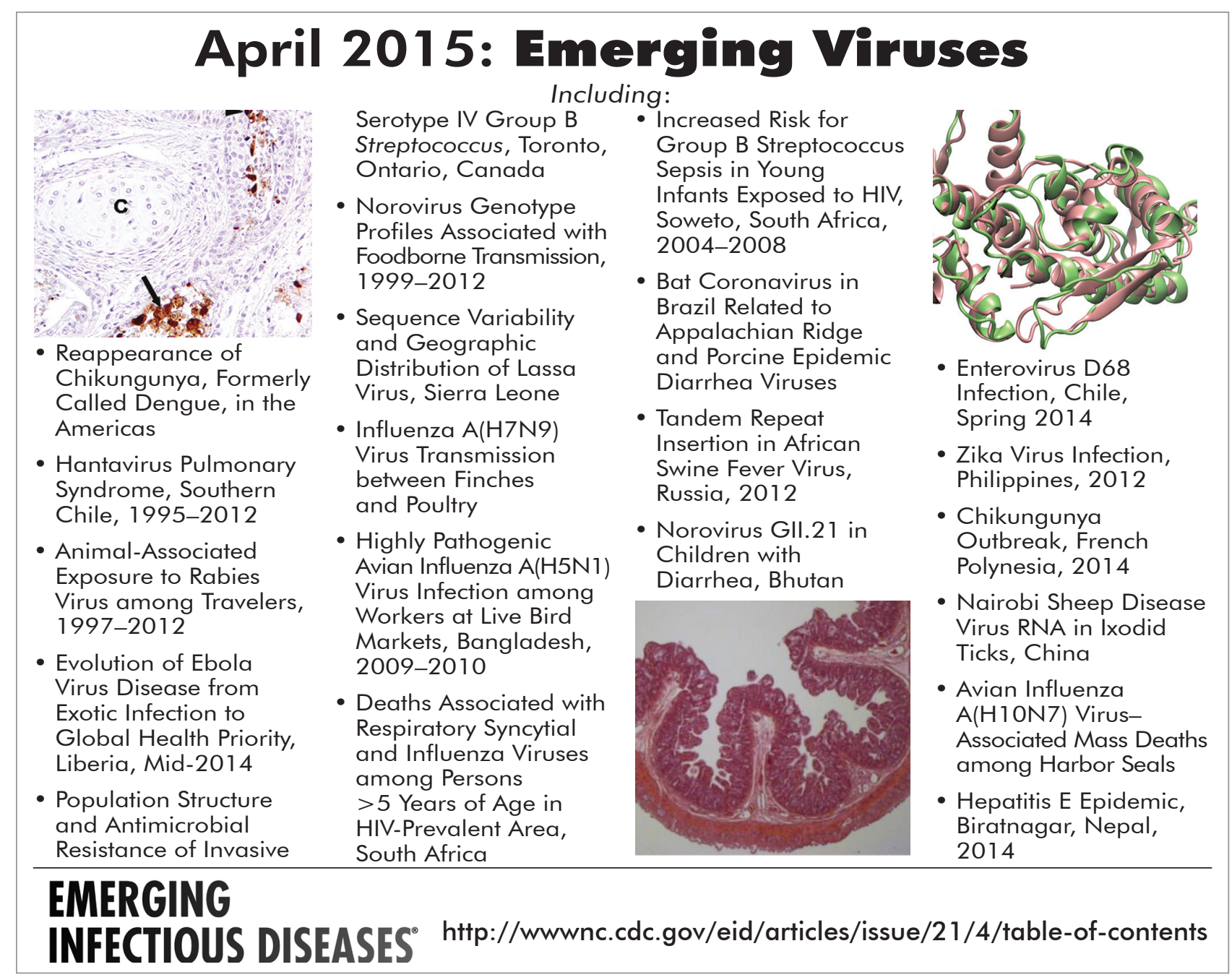

